\title{
UM RETRATO BIBLIOMÉTRICO DA PRODUÇÃO CIENTIIFICA BRASILEIRA SOBRE GESTÃO DA TECNOLOGIA E DA INOVAÇÃO NO PERÍODO 2001-2011
}

\author{
A BIBLIOMETRIC PORTRAIT OF THE BRAZILIAN \\ SCIENTIFIC PRODUCTION ON TECHNOLOGY AND \\ INNOVATION MANAGEMENT IN THE PERIOD 2001-2011
}

Recebido $19 / 11 / 2012$

Aceito 26/03/2013

Pedro Paulo de Andrade Júnior ${ }^{1}$

Fabrício Alexandre Alves Ceranto ${ }^{2}$

\section{RESUMO}

O presente trabalho teve como objetivo mapear a produção brasileira sobre Gestão da Tecnologia e da Inovação (GTI) no período 2001-2011, com vistas a identificar (i) o número de artigos publicados, (ii, iii, iv e v) periódicos, autores, instituições e estados que mais têm publicado sobre o assunto. O estudo se justifica pois a literatura tem apontado que o desenvolvimento de estudos na área de GTI é um fator que auxilia os países a alcançar maiores níveis de desempenho inovador, desde que estes conhecimentos sejam absorvidos pelos agentes nacionais responsáveis pelo fomento à inovação e ao desempenho tecnológico empresarial no país, e, também, pelas próprias empresas. Dessa maneira, o conhecimento fornecido nesse trabalho orienta sobre como a configuração da produção científica brasileira sobre GTI pode ser relevante, em diferentes aspectos, para agentes governamentais de fomento à inovação e para pesquisadores atuantes nessa área. O mapeamento foi realizado por meio de um método bibliométrico. Como resultado, obteve-se todos os aspectos do que se desejou levantar, ficando evidentes principalmente três características da produção científica brasileira sobre GTI: (i) um número não muito elevado de publicações; (ii) uma alta concentração institucional e territorial desse conhecimento tem sido produzido; e (iii) nas localidades onde há uma alta concentração da produção de conhecimento sobre GTI, os níveis de desenvolvimento tecnológico/inovador e socioeconômico se sobressaem em relação à média nacional, elucidando a relevância da produção científica sobre o assunto.

Palavras-chave: Gestão da Tecnologia e da Inovação, Mapeamento Bibliométrico, Políticas de C,T\&I, Desenvolvimento Tecnológico, Desenvolvimento Socioeconômico

${ }^{1}$ Possui graduação em Economia e mestrado e doutorado em Engenharia da Produção pela Universidade Federal de Santa Catarina - UFSC. Atualmente é professor adjunto na Universidade Tecnológica Federal do Paraná - UTFPR. Ponta Grossa, Paraná, Brasil. E-mail: pedropaulo@utfpr.edu.br.

${ }^{2}$ Atualmente é graduando em Engenharia Mecânica na Universidade Tecnológica Federal do Paraná - UTFPR. Ponta Grossa, Paraná, Brasil. E-mail: fabricioceranto@hotmail.com. 


\begin{abstract}
The present paper had as objective to map the Brazilian scientific production on Technology and Innovation Management (GTI) in the period 2001-2011, in view of identifying (i) the total number to published articles, as well as the number in each of the years; (ii, iii, iv and v) journals, authors, institutions and states that have most published articles. This study is justified because literature says that GTI studies may help countries to achieve higher levels of entrepreneurial innovation and technology performance, since these studies are absorbed by the companies and innovation incentive agencies. Thus, knowing how scientific production has been occurring in the country is an aspect of relevance for the governmental agencies that incentive entrepreneurial innovation in the country. This knowledge is also important for researchers, due to several other reasons. The mapping was made through a bibliometric method. The results show that the Brazilian scientific production on GTI is not very large, in terms of number, and it is too concentrated (in terms of institutions and territory), factors that certainly hinder the dissemination and rooting of an innovation culture in Brazil. From the results, it was also possible to verify that in the locations where GTI scientific production is more strongly concentrated the levels of technological, economic and social development are quite higher than the national average, which elucidates the mentioned relevance of the GTI scientific production.
\end{abstract}

Keywords: Technology and Innovation Management; Bibliometric Mapping; S,T\&I Policies; Technological Development; Socioeconomic Development

\title{
1 INTRODUÇÃO
}

Nos atuais paradigmas econômicos, de um modo geral, são os países cujas empresas têm apresentado maiores níveis de desempenho tecnológico e inovador no mercado mundial que têm conseguido obter maiores níveis de desenvolvimento socioeconômico (FLEURY; FLEURY, 1997; CARVALHO, 1999; MATTOS; GUIMARÃES; 2005; TIGRE, 2006; ANDREASSI, 2007; REIS, 2008). Isso ocorre porque essas empresas são capazes de atender à demanda interna do país e são fornecedoras globais em seus segmentos de atuação, de modo que causam um bastante efeito positivo na balança comercial do país (ROZENFELD et al., 2006).

Assim, o capital é acumulado pelas empresas e pode ser utilizado para proporcionar maior crescimento, mais emprego e mão de obra e, assim, maior distribuição de renda realizada pelo governo, muitas vezes na forma de tarifas de exportação, o qual pode se investir em várias áreas que favoreçam a qualidade de vida da população e/ou a manutenção do crescimento econômico do país (Saúde, Segurança, Educação, Infraestrutura, etc.) (FREEMAN; SOETE, 1997; TIGRE, 2006; CARPES et al., 2012).

Devido a isso, os governos de vários países têm realizado esforços na tentativa de promover um maior desempenho tecnológico e inovador para suas empresas, como a criação de agências governamentais de fomento financeiro à pesquisa acadêmica ou à inovação empresarial, a elaboração de políticas para promover maior integração entre instituições de pesquisa (como universidades) e empresas do país, entre outros aspectos (FREEMAN; SOETE, 1997; SIMANTOB; LIPPI, 2003; ANDREASSI, 2007; SILVA; GIULIANI, 2009).

Diante da relevância socioeconômica do assunto, essas medidas, provenientes do governo ou tomadas pelas próprias empresas, têm sido estudadas e, assim, tem se constituído um campo do conhecimento denominado Gestão da Tecnologia e da Inovação (GTI).

Defende-se nesse trabalho, com base nos trabalhos de Perruchas et al (2005), Junqueira e Mitre (2007) e Teixeira e Mota (2012), que o desenvolvimento de estudos na área de GTI auxilia os países a alcançar maiores níveis de desenvolvimento tecnológico e inovador, desde que as práticas ou conhecimentos defendidos nesses estudos sejam, de fato, absorvidos pelos agentes 
governamentais responsáveis pela inovação empresarial (quando são estudos que abordam a GTI em um nível de fomento governamental, por meio das agências, por exemplo) ou pelas próprias empresas (quando são estudos que abordam a GTI em um nível empresarial, por exemplo).

Dessa maneira, entender como a produção científica sobre a GTI tem ocorrido em um país é de relevância estratégica para agentes governamentais e pesquisadores, para, por exemplo, elaborar políticas científicas e tecnológicas no país. Para pesquisadores, esse conhecimento também é importante por elucidar quais têm sido os autores, instituições e periódicos que têm se destacado nas pesquisas sobre GTI e que, assim, podem ser visualizados como fontes ou parceiros de pesquisa (KNEIPP et al., 2011; TEIXEIRA; MOTA, 2012).

Assim, o presente trabalho tem o objetivo de mapear a produção científica brasileira sobre Gestão da Tecnologia e da Inovação (GTI) no período 2001-2011. Os seguintes aspectos, os quais que são de relevância para os agentes mencionados, foram mapeados: (i) número de artigos publicados no período, bem como em cada um dos anos; (ii, iii, iv e v) periódicos, autores, instituições e estados brasileiros que mais publicaram sobre o assunto no período compreendido. Tomaram-se como base para se realizar o mapeamento 51 periódicos científicos brasileiros, de acordo com um procedimento bibliométrico adotado descrito na seção Metodologia.

A seguir, é apresentada uma revisão de literatura sobre a relevância da produção científica sobre GTI sobre a obtenção de maiores níveis de desenvolvimento tecnológico e inovador nos países, pelo viés da disseminação desse conhecimento e da criação de uma cultura de inovação.

\section{A RELEVÂNCIA DA PRODUCCÃO CIENTÍFICA SOBRE GESTÃO DA TECNOLOGIA E DA INOVAÇÃO PARA O DESENVOLVIMENTO TECNOLÓGICO E INOVADOR DE REGIÕES E PAIISES}

\subsection{Relevância do conhecimento para o desenvolvimento dos países}

Vários estudos da literatura mundial têm apresentado as relações entre ciência, tecnologia/inovação e desenvolvimento econômico. A produção de conhecimento, sobretudo em áreas tecnológicas, permite a criação de novas tecnologias para atender demandas da sociedade, fato que, normalmente, gera resultados econômicos significativos (ALBUQUERQUE et al., 2002; ALBUQUERQUE et al., 2005; REIS, 2008; CARVALHO et al., 2011).

É um fluxo que se retroalimenta, uma vez que uma grande capacidade econômica também proporciona maiores investimentos em ciência ou Pesquisa \& Desenvolvimento (P\&D), ajudando a manter ou a elevar os resultados econômicos positivos que, ainda, podem auxiliar na promoção de desenvolvimento social (ALBUQUERQUE et al., 2002; ALBUQUERQUE et al., 2005; TIGRE, 2006).

Assim, a capacidade de produzir conhecimento científico de relevância e apropriar-se dele por meio da geração de inovações tem definido as regiões mais ricas e desenvolvidas dentro de um país e, também, os países mais ricos e desenvolvidos em relação aos demais (SCHUMPETER, 1988; DOSI, 1988; FREEMAN; SOETE, 1997; TIGRE, 2006; ANDREASSI, 2007).

\subsection{Relevância do conhecimento sobre Gestão da Tecnologia e da Inovação para o desenvolvimento dos países \\ Conforme apresentado na subseção anterior, o conhecimento tem apresentado grande importância para os países na obtenção de resultados econômicos positivos. \\ $\mathrm{O}$ conhecimento produzido em áreas gerenciais é de extrema importância para uma}


boa organização das empresas e outros agentes, mas, além disso, o conhecimento produzido em áreas tecnológicas também é de fundamental importância neste contexto econômico, pelo fato de que a partir deste podem ser geradas inovações. Entretanto, o processo de gerenciamento das interações entre conhecimento tecnológico produzido, criação de novas tecnologias e resultados econômicos é estudado por uma área gerencial específica, a Gestão da Tecnologia e da Inovação.

Assim, produzir conhecimento nessa área também tem se mostrado um aspecto de grande importância para que os países alcancem resultados econômicos significativos, pois é o emprego das boas práticas estudadas na GTT que permite que esse processo possa efetivamente ocorrer em um país (PERRUCHAS et al., 2005; JUNQUEIRA; MITRE, 2007; TEIXEIRA; MOTA, 2012).

Esse aspecto elucida a grande relevância de se produzir conhecimento na área de GTI e, além disso, gerar meios para que esse seja absorvido e praticado pelos agentes do sistema nacional de inovação.

\subsection{Bibliometria do conhecimento produzido sobre Gestão da Tecnologia e da Inovação}

Conhecer como os países têm produzido conhecimento nesta área tem-se tornado um aspecto estratégico para os agentes promotores de políticas científicas e tecnológicas dos países e para pesquisadores.

Quando a produção científica é baixa no país como um todo ou em regiões específicas, medidas (políticas) devem ser tomadas pelospromotores de políticas, com vistas a fomentar o enraizamento de uma cultura de inovação no país (PERRUCHAS et al., 2005; BIN, 2008; MARICATO, 2010).

Para os pesquisadores, conhecer a produção científica sobre GTI é relevante, pois eles podem auxiliar os agentes governamentais na elaboração de políticas, além de que passam a conhecer as instituições, os pesquisadores e os periódicos que têm se destacado na área e que podem ser visualizados como fonte ou parceiros de pesquisa (BIN, 2008; MARICATO, 2010).

Estudos que permitem obter esse tipo de conhecimento, isto é, sobre a produção científica em alguma área, são denominados estudos bibliométricos ou cientométricos (TAGUE-SUTCKIFFE, 1992; PRAT, 1998; GARFIELD, 1998; MUGNAINI; JANNUZZI; QUONIAM, 2004). Assim, destaca-se a importância da realização de estudos bibliométricos na área de GTI.

Apesar da pertinência do assunto, apenas alguns estudos bibliométricos na área de GTI têm sido encontrados na literatura, como os trabalhos de Perruchas et al. (2005), Junqueira e Mitre (2007) e Teixeira e Mota (2012); entretanto, nesses trabalhos, tal relevância é salientada.

\section{METODOLOGIA}

Conforme mencionado anteriormente, alinhando-se ao o objetivo do trabalho, adotouse um método bibliométrico que permitisse verificar como a produção científica brasileira sobre GTI tem se configurado diante dos seguintes aspectos: (i) quantidade de estudos publicados; (ii, iii, iv e v) quantidade de publicações por periódicos, autores, instituições e estados.

Para Holbrook (1992), ciência e tecnologia apresentam dimensões que podem ser medidas por indicadores, dos quais se esperam obter informações relevantes, não dedutíveis de forma trivial e compreensíveis para não acadêmicos.

Para que fosse possível realizar uma contabilização da produção científica sobre o assunto especificamente no Brasil, optou-se por não se utilizar o levantamento de artigos em bases de dados por meio de palavras-chaves relacionadas ao tema, pois esse método se mostra um tanto excludente da contabilização de artigos que têm sido desenvolvidos no âmbito nacional sobre 
o assunto, permitindo apenas uma visão quantitativa aproximada do crescimento percentual de publicações ao longo dos anos (REZENDE, 1990; ARAÚJO JÚNIOR; TARAPANOFF, 2006). Devido a ists, adotou-se como método de pesquisa o levantamento direto dos artigos nos portais eletrônicos dos periódicos nacionais que tratam do assunto.

Primeiramente, foi necessário definir quais seriam esses periódicos. De acordo com Tigre (2006), o assunto GTI tem sido estudado majoritariamente pelas áreas de Administração, Economia e Engenharia de Produção. Assim, foram tomados como base para a pesquisa os periódicos com estrato Webqualis superior a B5 em pelo menos uma dessas áreas, de acordo com a classificação da Capes (2010). Os periódicos que apresentam somente estratos B5 ou inexistentes nessas áreas foram considerados de pouca relevância científica nas áreas e, portanto, não foram tomados como base para o mapeamento. Adotando-se este critério de seleção, obtiveram-se 51 periódicos brasileiros que foram tomados como base para a pesquisa, os quais podem ser observados na Tabela 1 dos resultados desse trabalho.

Para, nesses periódicos, levantar os artigos referentes à GTI, um total de 105 palavraschaves (35 em português no singular, 35 em português no plural e 35 em inglês) foram sistematicamente lançadas na guia de pesquisa por artigos de cada periódico. Os resultados destas pesquisas apontavam uma série de artigos, que nem sempre eram estudos que traziam contribuições à GTI, de modo que seus títulos e - se ainda houvesse dúvidas quanto à abordagem seus resumos, foram lidos para levantar os artigos que realmente fossem pertinentes à GTI.

Estes artigos levantados foram salvos na plataforma computacional utilizada na pesquisa e, posteriormente, seus dados referentes à autoria (periódico, autores, instituição e estado) foram coletados e sistematicamente lançados em um banco de dados, o Microsoft Excel ${ }^{\circledR}$, para classificação e, assim, elaboração dos resultados.

Os resultados obtidos são apresentados na próxima seção.

\section{RESULTADOS}

Nesta seção, são apresentados os resultados do mapeamento da produção científica brasileira sobre GTI no período 2001-2011. Os diferentes aspectos levantados são apresentados em diferentes subseções.

\subsection{Número de artigos publicados no período}

O mapeamento bibliográfico permite uma visualização com certa precisão da produção científica brasileira sobre GTI; apontou-se um número total de 508 artigos publicados sobre o assunto entre 2001 e 2011. Além disso, também foi possível verificar a quantidade de artigos publicados em cada um dos anos do período analisado, conforme mostrado na Figura 1. 


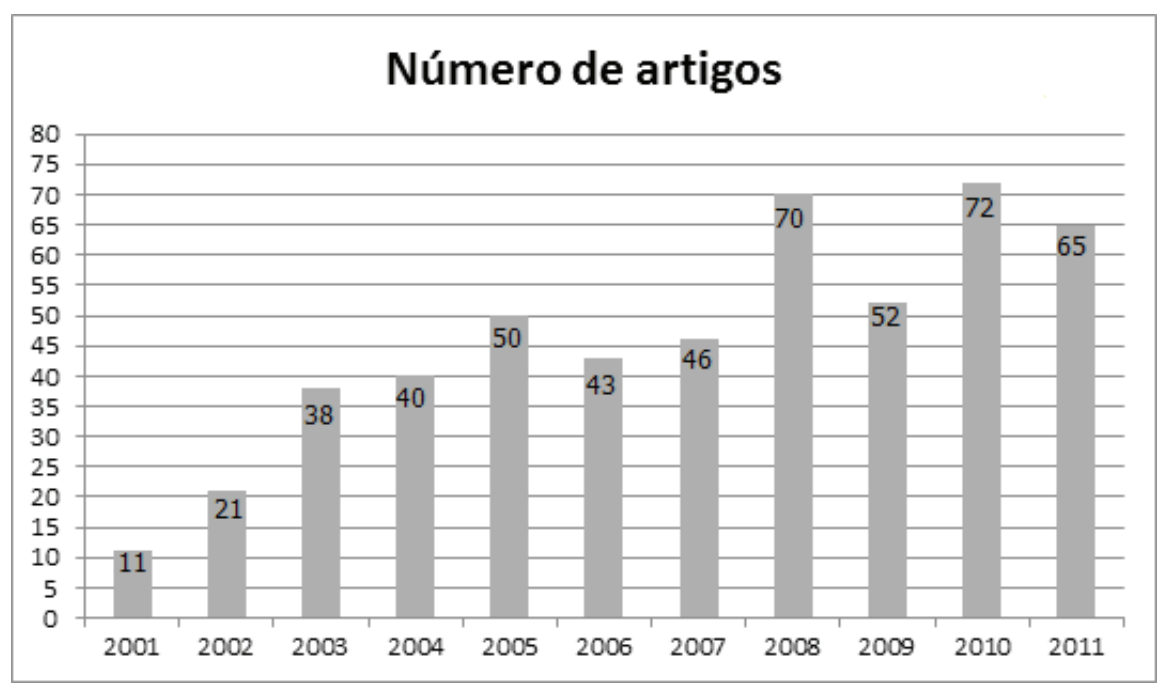

Figura 1 - Número de artigos publicados sobre Gestão da Tecnologia e da Inovação entre 2001 e 2011 nos 51 periódicos brasileiros tomados como base para a pesquisa

Observa-se, de um modo geral, o crescente número de publicações (com exceção dos anos de 2005, 2008 e 2010, que superaram seus anos subsequentes no número de artigos), assim como tem ocorrido na maior parte das áreas do conhecimento. Da média de 23 artigos por ano nos três primeiros anos do período analisado, houve um aumento para uma média de 63 artigos por ano nos três últimos anos.

\subsection{Quantidade de artigos publicados em cada periódico}

A Revista Brasileira de Inovação (RBI) e a Revista de Administração e Inovação (RAI) foram os dois periódicos que mais publicaram estudos sobre o assunto no período observado, o que se deve ao fato de serem os dois periódicos (dentre os 51 tomados como base para o mapeamento que declararam em suas políticas editoriais o interesse exclusivo pela divulgação de estudos que sejam relativos a temas de áreas da Gestão da Tecnologia e da Inovação.

A lista do número de artigos publicados sobre GTI entre 2001 e 2011 em cada um dos periódicos tomados como base para o levantamento é mostrada na Tabela 1.

\begin{tabular}{|c|c|c|c|}
\hline Periódico & $\begin{array}{l}\text { Número } \\
\text { de artigos }\end{array}$ & Periódico & $\begin{array}{l}\text { Número } \\
\text { de artigos }\end{array}$ \\
\hline $\begin{array}{c}\text { Revista Brasileira de Inovação (UNICAMP, } \\
\text { FINEP) }\end{array}$ & 85 & $\begin{array}{l}\text { Revista de Estudos Econômicos } \\
\text { (USP) }\end{array}$ & 6 \\
\hline $\begin{array}{c}\text { Revista de Administração e Inovação (RAI) } \\
\text { (Várias instituições) }\end{array}$ & 46 & $\begin{array}{c}\text { Revista de Administração Pública } \\
\text { - RAP }\end{array}$ & 5 \\
\hline $\begin{array}{l}\text { Read. Revista Eletrônica de Administração } \\
\text { (UFRGS) }\end{array}$ & 32 & Análise Econômica (UFRGS) & 5 \\
\hline Revista Ciências Administrativas & 26 & Revista de Administração FACES & 5 \\
\hline $\begin{array}{c}\text { Revista Gestão \& Tecnologia (Fundação Pe- } \\
\text { dro Leopoldo) }\end{array}$ & 25 & Estudos econômicos (FEA-USP) & 5 \\
\hline Gestão \& Produção (UFSCar) & 21 & $\begin{array}{l}\text { RACE. Revista de Administração, } \\
\text { Contabilidade e Economia }\end{array}$ & 5 \\
\hline Produção Online (UFSC) & 15 & $\begin{array}{l}\text { Revista Brasileira de Estudos Re- } \\
\text { gionais e Urbanos (ABER) }\end{array}$ & 5 \\
\hline
\end{tabular}




\begin{tabular}{|c|c|c|c|}
\hline Periódico & $\begin{array}{l}\text { Número } \\
\text { de artigos }\end{array}$ & Periódico & $\begin{array}{l}\text { Número } \\
\text { de artigos }\end{array}$ \\
\hline $\begin{array}{c}\text { Revista Gestão Industrial (UTFPR Campus } \\
\text { Ponta Grossa) }\end{array}$ & 15 & Gestão.Org (UFPE) & 3 \\
\hline Produção (USP) & 14 & $\begin{array}{c}\text { Economia Aplicada (USP Ribeirão } \\
\text { Preto) }\end{array}$ & 3 \\
\hline $\begin{array}{c}\text { Revista Brasileira de Gestão e Desenvolvi- } \\
\text { mento Regional }\end{array}$ & 14 & $\begin{array}{c}\text { Revista de Administração da FEAD } \\
\text { - Minas }\end{array}$ & 3 \\
\hline $\begin{array}{l}\text { Revista de Administração Contemporânea } \\
\text { (ANPAD) }\end{array}$ & 14 & Revista Econômica do Nordeste & 3 \\
\hline $\begin{array}{l}\text { RAUSP-e/Revista de Administração da USP } \\
\text { (USP) }\end{array}$ & 13 & $\begin{array}{c}\text { Revista Brasileira de Gestão de } \\
\text { Negócios }\end{array}$ & 2 \\
\hline Revista de Ciências da Administração & 12 & Revista Produto \& Produção & 2 \\
\hline Revista de Negócios (FURB) & 12 & Revista de economia política & 2 \\
\hline Revista Economia \& Gestão (PUC-MG) & 10 & Nova Economia (UFMG) & 2 \\
\hline Revista de Administração de Empresas & 9 & $\begin{array}{l}\text { Revista de Economia e Adminis- } \\
\text { tração }\end{array}$ & 2 \\
\hline Revista de Administração Mackenzie & 9 & $\begin{array}{l}\text { Revista Brasileira de Economia } \\
\text { (FGV) }\end{array}$ & 1 \\
\hline BrazilianAdministrationReview & 9 & $\begin{array}{l}\text { Revista de Estudos de Adminis- } \\
\text { tração }\end{array}$ & 1 \\
\hline $\begin{array}{l}\text { Revista de Desenvolvimento Econômico } \\
\text { (UNIFACS - Salvador) }\end{array}$ & 9 & $\begin{array}{c}\text { Revista Brasileira de Economia de } \\
\text { Empresas (UCB) }\end{array}$ & 1 \\
\hline Revista de Economia (UFPR) & 9 & $\begin{array}{c}\text { Revista Eletrônica Produção \& } \\
\text { Engenharia }\end{array}$ & 1 \\
\hline Econômica (UFF Niterói) & 9 & Política e Sociedade (UFSC) & 1 \\
\hline Organizações \& Sociedade (UFBA) & 8 & Gestão \& Planejamento & 0 \\
\hline Economia e Sociedade (UNICAMP) & 8 & Brazilian Business Review & 0 \\
\hline REGE. Revista de Gestão USP (USP) & 7 & $\begin{array}{l}\text { Revista de Políticas Públicas } \\
\text { (UFMA) }\end{array}$ & 0 \\
\hline $\begin{array}{l}\text { Revista de Economia Contemporânea } \\
\text { (UFRJ) }\end{array}$ & 7 & $\begin{array}{c}\text { Revista Brasileira de Estudos Ur- } \\
\text { banos e Regionais (ANPUR) }\end{array}$ & 0 \\
\hline $\begin{array}{l}\text { Revista Portuguesa e Brasileira de Gestão } \\
\text { (ISCTE-Portugal, FGV) }\end{array}$ & 7 & & \\
\hline
\end{tabular}

\subsection{Quantidade de artigos publicados por cada autor}

A lista dos autores que mais publicaram sobre o assunto (4 artigos ou mais) é mostrada na Tabela 2. A lista completa pode ser obtida com os autores via e-mail.

\begin{tabular}{ccc}
\hline Autor & Instituição de origem do autor & Número de artigos publicados \\
\hline Paulo Negreiros Figueiredo & FGV-RJ & 11 \\
Andréa Paula Mendes Segatto & UFPR & 8 \\
Eduardo Gonçalves & UFMG & 7 \\
Uma Urraca Ruiz & UFF & 6 \\
Geciane Silveira Porto & USP São Paulo & 6
\end{tabular}




\begin{tabular}{|c|c|c|}
\hline Autor & Instituição de origem do autor & Número de artigos publicados \\
\hline Renato Dagnino & UNICAMP & 6 \\
\hline Aline França Abreu & UFSC & 5 \\
\hline Ana Lúcia Vitale Torkomian & UFSCar & 5 \\
\hline Eduardo da Motta e Albuquerque & UFMG & 5 \\
\hline João Luiz Kovaleski & UTFPR Ponta Grossa & 5 \\
\hline Marcelo Seido Nagano & USP São Carlos & 5 \\
\hline Milton de Abreu Campanário & UNINOVE & 5 \\
\hline Roberto Sbragia & USP São Paulo & 5 \\
\hline Wilson Suzigan & UNICAMP & 5 \\
\hline Dálcio Roberto dos Reis & $\begin{array}{c}\text { UTFPR Ponta Grossa/UTFPR } \\
\text { Curitiba }\end{array}$ & 4 \\
\hline Daniel Capaldo Amaral & USP São Carlos & 4 \\
\hline Eva Stal & UNINOVE & 4 \\
\hline Fabiano Maury Raupp & UDESC & 4 \\
\hline Francisco Lima Cruz Texeira & UFBA & 4 \\
\hline Isabel Cristina dos Santos & UNITAU & 4 \\
\hline José Vitor Bomtempo & UFRJ & 4 \\
\hline Luiz Paulo Bignetti & UNISINOS & 4 \\
\hline Marta Araújo Tavares Ferreira & UFMG & 4 \\
\hline Paulo Bastos Tigre & UFRJ & 4 \\
\hline Paulo Roberto Tavares Dalcol & PUC-RJ & 4 \\
\hline Sieglinde Kindle Cunha & PUC-RJ & 4 \\
\hline Simone Vasconcelos Ribeira Galina & USP Ribeirão Preto & 4 \\
\hline
\end{tabular}

Tabela 2 - Autores que mais publicaram sobre Gestão da Tecnologia e da Inovação entre 2001 e 2011 nos 51 periódicos brasileiros tomados como base para a pesquisa

Fonte: Elaborado pelos autores

Na obtenção dos resultados apresentados na Tabela 2, quando da existência de dois ou mais autores por artigo, o valor de um artigo foi contabilizado para cada um dos autores.

\subsection{Quantidade de artigos publicados por cada instituição}

Observando-se as instituições de filiação de cada autor, as quais constam nos artigos, foi possível listar as instituições nas quais os artigos foram desenvolvidos. As 42 instituições que mais contribuíram com o desenvolvimento de artigos (4 artigos ou mais) estão listadas na Tabela 3. A lista completa pode ser obtida com os autores via e-mail.

\begin{tabular}{cc|cc}
\hline Instituição & Número de artigos & Instituição & Número de artigos \\
\hline USP & 74 & Embrapa & 6 \\
UFRJ & 29 & UFLA & 6 \\
UNICAMP & 27 & UFPB & 6 \\
UFMG & 25 & UFV & 6 \\
UFPR & 25 & UNITAU & 6 \\
FGV & 24 & Fundação Pedro Leopoldo & 5 \\
UFRGS & 20 & Ipea & 5 \\
UFSC & 19 & PUC-RS & 5 \\
UFSCar & 17 & UEM & 5
\end{tabular}




\begin{tabular}{cc|cc}
\hline Instituição & Número de artigos & Instituição & Número de artigos \\
\hline UNINOVE & 15 & UFCE & 5 \\
UNISINOS & 14 & UNIFOR & 5 \\
UFF & 12 & Fundação João Pinheiro & 4 \\
UFPE & 11 & INPI & 4 \\
FURB & 10 & IPT & 4 \\
UTFPR & 10 & Mackenzie & 4 \\
PUC-MG & 9 & Petrobras & 4 \\
UFBA & 9 & PUC-RJ & 4 \\
UFJF & 9 & UECE & 4 \\
UFSM & 8 & UFCG & 4 \\
UFU & 8 & UNIFACS (Salvador) & 4 \\
UnB & 8 & Universidade Positivo (UP) & 4 \\
\hline
\end{tabular}

Tabela 3 - Instituições que mais publicaram sobre Gestão da Tecnologia e da Inovação entre 2001 e 2011 nos 51 periódicos brasileiros tomados como base para a pesquisa

Fonte: Elaborado pelos autores

Para realizar tal contabilização, utilizou-se como método somar um artigo publicado a cada instituição, desde que um dos autores do artigo fosse proveniente de tal instituição. Assim, cada artigo com autores de diferentes instituições, contabilizou uma publicação para cada uma das instituições.

\subsection{Quantidade de artigos desenvolvidos por cada unidade federativa do país}

Com base na contabilização de artigos publicados por cada instituição pôde-se também verificar a quantidade de artigos publicados por cada estado, conforme mostrado na Tabela 4.

\begin{tabular}{ccc|cc|c}
\hline Estado & $\begin{array}{c}\text { Número de } \\
\text { artigos }\end{array}$ & $\begin{array}{c}\text { Porcentagem em } \\
\text { relação ao núme- } \\
\text { ro total de artigos }\end{array}$ & Estado & $\begin{array}{c}\text { Número de } \\
\text { artigos }\end{array}$ & $\begin{array}{c}\text { Porcentagem em } \\
\text { relação ao número } \\
\text { total de artigos }\end{array}$ \\
\hline São Paulo & 190 & $33,57 \%$ & Ceará & 14 & $2,47 \%$ \\
Minas Gerais & 97 & $17,14 \%$ & Paraíba & 10 & $1,77 \%$ \\
Rio de Janeiro & 69 & $12,19 \%$ & Distrito Federal & 8 & $1,41 \%$ \\
Paraná & 53 & $9,36 \%$ & Espírito Santo & 3 & $0,53 \%$ \\
Rio Grande do Sul & 47 & $8,3 \%$ & Alagoas & 2 & $0,35 \%$ \\
Santa Catarina & 37 & $6,54 \%$ & Goiás & 2 & $0,35 \%$ \\
Bahia & 15 & $2,65 \%$ & Pará & 2 & $0,35 \%$ \\
Pernambuco & 15 & $2,65 \%$ & Sergipe & 2 & $0,35 \%$ \\
\hline
\end{tabular}

Tabela 4 - Número de artigos publicados sobre Gestão da Tecnologia e da Inovação entre 2001 e 2011 em cada unidade federativa e porcentagem em relação do país ao número total de artigos

Fonte: Elaborado pelos autores

No caso dos artigos que são de autorias de instituições de diferentes estados, foi contabilizado um artigo publicado para cada estado da autoria, de modo que o somatório do número de artigos publicados por cada estado da Tabela 4 não será igual ao número total de artigos, isto é, 508 (será maior).

Dos dados da Tabela 4, analisando-se os resultados por região, obtêm-se a distribuição apresentada na Tabela 5. 


\begin{tabular}{ccc}
\hline Estado & Número de artigos & $\begin{array}{c}\text { Porcentagem do número total de } \\
\text { artigos }\end{array}$ \\
\hline Sudeste & 359 & $63,43 \%$ \\
Sul & 137 & $24,20 \%$ \\
Nordeste & 58 & $10,25 \%$ \\
Centro-Oeste & 10 & $1,77 \%$ \\
Norte & 2 & $0,35 \%$ \\
\hline
\end{tabular}

Tabela 5 - Número de artigos publicados sobre Gestão da Tecnologia e da Inovação entre 2001 e 2011 em cada região do país e porcentagem em relação ao número total e artigos publicados no país

Fonte: Elaborado pelos autores

\section{CONSIDERAÇÕES FINAIS}

Do mapeamento da produção científica brasileira sobre Gestão da Tecnologia e da Inovação no período 2001-2011, foi possível observar principalmente três aspectos de grande importância, que devem ser mencionados.

Primeiramente, observou-se, diante do tamanho do país - em termos de extensão territorial ou número de habitantes -, um pequeno número de artigos publicados sobre o assunto GTI: 46,18 artigos por ano ou 0,9055 artigos por periódico por ano.

Em segundo lugar, observa-se que a produção científica brasileira sobre GTI encontra-se fortemente concentrada em determinados estados (conforme analise da seção 4.5) e também em municípios (conforme se pode extrair da análise de publicações por instituições).

Acredita-se, com base no apresentado na literatura, que o fato da pequena produção científica brasileira sobre GTI, estando concentrada majoritariamente em alguns municípios do país, é um fator que dificulta a disseminação de uma cultura de inovação no país.

Apesar desse número de publiçções considerado não muito alto, a terceira observação foi de que, nos municípios (em que se localizam as instituições) onde houve uma maior produção científica sobre GTI no período estudado, há um destaque no cenário nacional pelo desenvolvimento tecnológico e socioeconômico. Isto coaduna com as relações entre ciência, tecnologia e desenvolvimento que foram apresentadas na revisão de literatura.

Acredita-se que são estudos que, se levados em consideração pelos agentes do sistema nacional de inovação, podem trazer contribuições significativas para o cenário tecnológico nacional. Desta maneira, sugere-se neste trabalho, a necessidade de políticas governamentais para incentivar uma maior produção científica na área de GTI, no país como um todo, sobretudo nas localidades nas quais esta ainda se mostra muito baixa.

Além disso, para que, de fato, o desenvolvimento tecnológico possa ocorrer, sugere-se a necessidade de políticas governamentais para que, de alguma maneira, esses conhecimentos sobre GTI sejam de fato absorvidos pelos agentes do SNI, dentre os quais se enquadram empresas, universidades e o próprio governo. Comentários sobre a baixa absorção do conhecimento científico no meio empresarial brasileiro podem ser encontrados nos trabalhos de Tabet (2001), Alvim (2007) e Silva (2009).

Alerta-se para o fato de que muitas das contribuições trazidas pela literatura dessa área, como a do presente trabalho, são relativas a medidas de melhoria que devem ser executadas pelo governo. portanto o próprio governo deve ser um agente que esteja atento para o conhecimento científico gerado. Para um maior aprofundamento, esta é a questão abordada no trabalho de Schawartzman (2002). 
Nota dos autores: Na elaboração desse trabalho, foram empregados muito zelo e técnica, bem como compromisso com a veracidade da teoria apresentada, do conteúdo citado e dos resultados obtidos.

\section{REFERÊNCIAS}

ALBUQUERQUE, E. M.; SIMÕES, R.; BAESSA, A.; CAMPOLINA, B.; SILVA, L. A distribuição espacial da produção científica e tecnológica brasileira: uma descrição de estatísticas de produção local de patentes e artigos científicos. Revista Brasileira de Inovação, v. 1, n. 2, Julho/ Dezembro de 2002.

ALBUQUERQUE, E. M.; BAESSA, A. R.; KIRDEIKAS, J. C. V.; SILVA, L. A.; RUIZ, R. $M$. Produção científica e tecnológica nas regiões metropolitanas brasileiras. Revista de Economia Contemporânea, Rio de Janeiro, v. 9, n. 3, Setembro/Dezembro de 2005.

ALVIM, P. C. R. Gestão do Conhecimento nas empresas de pequeno porte. In: Anais do II Workshop de Inteligência Competitiva e Gestão do Conhecimento. 2001.

ANDREASSI, T. Gestão da Inovação Tecnológica. São Paulo: Thomson Learning, 2007. (Coleção Debates em Administração. VASCONCELOS, I. F. G.; VASCONCELOS, F. C. (Coord.)).

ARAÚJO JÚNIOR, R. H.; TARAPANOFF, K. Precisão no processo de busca e recuperação da informação: uso da mineração de textos. Revista Ciência da Informação, Brasília, v. 35, n. 3, p. 236-247, set./dez. de 2006.

BIN, A. Planejamento e Gestão da Pesquisa e da Inovação. 2008. 253 f. Tese (Doutorado em Política Científica e Tecnológica). Instituto de Geociência da Universidade Estadual de Campinas - UNICAMP, 2008.

CAPES-COORDENAÇÃODEAPERFEIÇOAMENTO DE PESSOAL DE NÍVEL SUPERIOR. WebQualis. 2010. Disponível em <http://qualis.capes.gov. $\mathrm{br} /$ webqualis/>. Acesso em 12 de janeiro de 2012.
CARPES, A. M.; SCHERER, F. L.; BEURON, T. A.; SANTOS, M. B.; BORGES, D. E.; CARVALHO, B. M. Revista de Administração da UFSM, Santa Maria, v.5, n.2, p. 321-335, Mai./Ago. de 2012.

CARVALHO, A. V. Aprendizagem organizacional: em tempos de mudança. São Paulo: Pioneira, 1999.

CARVALHO, H. G.; REIS, D. R.; CAVALCANTE, M. B. Gestão da Inovação. 1. ed. Curitiba - Paraná: Aymará, 2011. v. 1. 136p.

DOSI, G. Technological change and economic theory. Londres: Pinter Publishers, 1988.

FLEURY, A. C. C.; FLEURY, M. T. L. Aprendizagem e inovação organizacional: as experiências de Japão, Coréia e Brasil. 2. Ed. São Paulo: Atlas, 1997. 237 p.

FREEMAN, C.; SOETE, L.The economics of industrial innovation. 3. Ed. Londres/ Washington: Pinter Publishers, 1997.

HOLBROOK, J. A. D. Why measure science? Science and Public Policy, v. 19, n. 5, p. 262266, Outubro de 1992.

GARFIELD, E. Quantitative analysis of the scientific literature and itsimplications for science policymaking in Latin America and the Caribbean. Bulletin of PAHO, v. 29, n. 1, p. 8795, 1995.

JUNQUEIRA, B.; MITRE, M. Value of bibliometric analysis for research policy: a case study of Spanish research into innovation and technology management. Scientometrics, Vol. 71, No. 3, 2007, pp. 443-454.

KNEIPP, J. M.; ROSA, L. A. B.; BICHUETI, R. S.; MADRUGA, L. R. R. C; JÚNIOR, V. F. S. Emergência da temática da Inovação Sustentável: uma análise da produção científica através da base Web of Science. Revista de Administração da 
UFSM, Santa Maria, v. 4, n. 3, p. 442-457, Set/ Dez. de 2011.

MARICATO, J. M. Dinâmica das relações entre ciência e tecnologia: um estudo bibliométrico e cientométrico de múltiplos indicadores de artigos e patentes em biodiesel. 2010. Tese (Doutorado em Ciência da Informação). PPGCI da Universidade de São Paulo (USP).

MATTOS, J. R. L.; GUIMARÃES, L. S. Gestão da Tecnologia e da Inovação: uma abordagem prática. São Paulo: Saraiva, 2005.

MUGNAINI, R.; JANNUZZI, P. M.; QUONIAM, L. Indicadores bibliométricos da produção científica brasileira. Ciência da Informação, Brasília, v. 33, n. 2, p. 123-131, maio/ago. 2004.

PERRUCHAS, F.; YEGROS-YEGROS, A.; MARTINEZ, E. C.; DE LUCIO, I. F. La investigación sobre "Sistemas de innovación": radiografía realizada a través del análisis de las publicaciones científicas en bases de datos internacionales. Revista Ciências Administrativas, Fortaleza, v. 11, n. 1, p. 51-63, ago. 2005.

PRAT, A. M. Avaliação da produção científica como instrumento para o desenvolvimento da ciência e da tecnologia. Ciência da Informação, Brasília, v. 27, n. 2, p. 206-209, maio/ago. 1998.

REIS, D. R. Gestão da Inovação Tecnológica. 2. Ed. Barueri: Manole, 2008.

REZENDE, Y. Análise comparativa do custo de pesquisas bibliográficas em abstracts impressos e em bases de dados online. Revista Ciência da Informação, Brasília, v. 19, n. 1, jan./jun. de 1990.

ROZENFELD, H; FORCELLINI, F. A.; AMARAL, D. C.; TOLEDO, J. C.; SILVA, S. L; ALLIPRADINI, D. H.; SCALICE, R. K. Gestão de desenvolvimento de produtos: uma referência para a melhoria do processo. São Paulo: Saraiva, 2006.

SCHAWARTZMAN, S. A pesquisa científica e o interesse público. Revista Brasileira de
Inovação, v. 1, n. 2, Julho/Dezembro de 2002.

SCHUMPETER, J. Teoria do desenvolvimento econômico, 1911. Edição Brasileira. São Paulo: Editora Nova Cultural, 1988.

SIMANTOB, M.; LIPPI, R. Guia Valor Econômico de Inovação nas Empresas. São Paulo: Globo, 2003.

SILVA, N. C. D.; GIULIANI, A. C. Um estudo sobre o desenvolvimento no Brasil da cooperação universidade-empresa - interação entre a instituição de ensino superior de tecnologia e a micro e pequena empresa. Revista de Administração da UFSM, Santa Maria, v. 2, n. 3, p. 479-498, Set./ Dez. de 2009.

SILVA, A. M. Impactos da geração e absorção de conhecimento na produtividade da firma. Revista de Economia Contemporânea. Rio de Janeiro, v. 13 , n. 3, pp. 467-487, Setembro/ Dezembro de 2009.

TABET, M. B. Transferência de conhecimento no contexto da globalização de empresas. 96 f. 2007. Dissertação (Mestrado profissional em Administração. Faculdade de Economia e Finanças IBMEC. 2007.

TAGUE-SUTCKIFFE, J. An introduction to informetrics. InformationProcessing\& Management, v. 28, n. 1, p. 1-3, 1992.

TEIXEIRA, A. A. C.; MOTA, L. (2012). A bibliometric portrait of the evaluation, scientific roots and influence of the literature on university-industry links. Scientometrics, v. 92, $25 \mathrm{p}$.

TIGRE, P. B. Gestão da Inovação: a economia da tecnologia no Brasil. Rio de Janeiro: Elsevier, 2006. 